\title{
The Numerical Solution of Hyperbolic Systems Using Bicharacteristics
}

\author{
By R. L. Johnston and S. K. Pal*
}

\begin{abstract}
This paper is concerned with deriving methods for solving numerically a firstorder quasilinear hyperbolic system. The basic principle of the method is to integrate along one of the bicharacteristics of the system to obtain an equivalent integral system. The numerical methods are then obtained by making suitable approximations to this integral system. Stability and convergence properties are analyzed in some detail. The methods are relatively easy to implement and have been successfully applied to problems in one, two and three space dimensions in such areas as magnetohydrodynamics and dynamic elasticity.
\end{abstract}

1. Introduction. In this paper, we shall deal with the problem of solving numerically the first-order hyperbolic system of partial differential equations

$$
\frac{\partial U}{\partial t}+\sum_{j=1}^{m} A_{i} \frac{\partial U}{\partial x_{i}}=0, \quad t>0
$$

where $x=\left(x_{1}, \cdots, x_{m}\right)$ is a point in Euclidean $m$-space $E^{n}$. Here, $U=\left(u_{1}, \cdots, u_{N}\right)^{T}$, where $u_{i}=u_{i}\left(t, x_{1}, \cdots, x_{m}\right), 1 \leqq i \leqq N$, are the dependent variables and each $A_{i}$ is an $N \times N$ matrix. The elements of the $A_{i}$ 's can be functions of the dependent as well as the independent variables but we shall assume they have continuous partial derivatives, are uniformly bounded and satisfy a uniform Lipschitz condition with respect to all variables. We shall solve the system (1.1) subject to the initial conditions

$$
U(0, x)=G(x) .
$$

We shall also assume that the problem is properly posed [14, p. 63]. We point out that our methods can be applied equally well to inhomogeneous problems although, for convenience, we shall discuss homogeneous problems only.

For problems in one space dimension $(m=1)$ a number of good methods are available including that of Courant, Isaacson and Rees [3] and the method of characteristics as described, for example, in [13]. These methods make use of special properties of the solution $U(t, x)$ along the characteristic curves. For problems where $m=2$, there are the methods of Lax and Wendroff [10] and [11]. Strang [15] has also proposed a method which is quite good. The method of characteristics can be extended but, even for $m=2$ ([1] and [6]), the resulting algorithm is quite complicated because considerable effort has to be expended in determining in which direction one should integrate along the characteristic surface. Butler [2] suggested a method

Received April 20, 1971.

AMS 1970 subject classifications. Primary 65M25, 65M05; Secondary 65M10. gence.

Key words and phrases. Bicharacteristics, finite difference, hyperbolic system, stability, conver-

* This research was sponsored in part by the National Research Council of Canada under grant number NRC A5262. 
which makes use of the bicharacteristics of the system (1.1) but, in addition to the great deal of computation involved, the derivation of the characteristic equations and elimination of the unknown derivatives by taking suitable linear combinations is quite a formidable task.

The purpose of this paper is to propose a new way of deriving finite difference schemes for solving (1.1). The basic principle involves integrating along a suitable bicharacteristic of the system to obtain an equivalent integral system. Difference schemes of arbitrary accuracy can then be obtained by making suitable approximations to the latter. One advantage of our approach is that it is independent of the number of space variables. In this paper, we shall confine our attention to two firstorder schemes derived by this method. While the accuracy is low, we shall see that they are relatively easy to implement and are computationally feasible even for problems in three space dimensions.

2. An Equivalent Integral System. In order to obtain an integral system equivalent to (1.1), we shall make use of the bicharacteristics of (1.1). We first give a brief description of the derivation of the equations of the bicharacteristics. A more detailed exposition is given in [7, p. 40] or [4, p. 200].

A characteristic surface $S$ of the system (1.1) is defined to be a generalized surface or manifold in $(m+1)$-dimensional space-time across which there is a jump in the normal derivative of the solution $U$ of (1.1). We denote such a surface by

$$
\phi\left(t, x_{1}, \cdots, x_{m}\right)=0
$$

and introduce new coordinates $\xi_{0}, \xi_{1}, \cdots, \xi_{m}$, where $\xi_{0}=\phi$, so that $\xi_{0}=0$ is the surface $S$ itself and $U_{\xi_{0}}$ is the derivative of $U$ in the direction normal to $S$. Hence, $S$ is characteristic if the coefficient of $U_{\xi_{0}}$ in the transformed version of (1.1) is singular, i.e., if

$$
H=\operatorname{det}\left(\lambda I+\sum_{i=1}^{m} \lambda_{i} A_{i}\right)=0,
$$

where $\lambda=\phi_{t}$ and $\lambda_{i}=\phi_{x_{i}}, 1 \leqq j \leqq m$. This is a first-order partial differential equation of degree $N$ for $\phi$. Since (1.1) is hyperbolic, $H$ can be written as the product of $N$ linear factors,

$$
H=\prod_{k=1}^{N}\left(\lambda \dot{+} h_{k}\right)
$$

where $h_{k}=h_{k}\left(\lambda_{1}, \cdots, \lambda_{m}, t, x_{1}, \cdots, x_{m}, u_{1}, \cdots, u_{N}\right)$ are the (real) eigenvalues of $\sum A_{i} \lambda_{i}$. Hence, $\phi$ is completely determined by the $N$ equations

$$
\lambda+h_{k}=0, \quad 1 \leqq k \leqq N .
$$

Now the characteristic curves of, say, the $k$ th equation of (2.3) can be specified $[4$, p. 201] by

$$
\begin{array}{cc}
d t / d s=1, & \\
d x_{i} / d s=\partial h_{k} / \partial \lambda_{i}, & 1 \leqq i \leqq m, \\
d \lambda_{i} / d s=-\partial h_{k} / \partial x_{i}, & 1 \leqq i \leqq m,
\end{array}
$$

where $d s$ represents a line element on $S$. Thus, corresponding to the $N$ equations 
(2.3), we have the $N$ systems of characteristic curves known as the bicharacteristics of (1.1). These bicharacteristics can be shown to be everywhere tangential to $S$ and, indeed, are generators of $S$. If $C$ is one such bicharacteristic, we can, by virtue of (2.4), denote differentiation along $C$ by $d / d t$. Hence, with (2.5), we have

or, using (1.1),

$$
d U / d t=U_{t}+\sum_{i=1}^{m} U_{x}, d x_{i} / d t
$$

$$
\frac{d U}{d t}=\sum_{i=1}^{m}\left(\frac{\partial h}{\partial \lambda_{i}} I-A_{i}\right) U_{x_{i}},
$$

where, for convenience, we have dropped the subscript $k$ denoting the particular eigenvalue $h_{k}$ giving rise to $C$. In what follows, this convention of using $h$ to denote one of the $h_{k}$ 's will be used.

Now, suppose $U$ is known at all points $(t, x), 0 \leqq t \leqq \tau$, and all $x$, and we want to find $U$ at the point $P(\tau+\Delta t, \alpha)$, where $\alpha=\left(\alpha_{1}, \cdots, \alpha_{m}\right) \in E^{m}$. Let $C$ be some bicharacteristic through $P$, and $S(\tau, \beta)$ be the point where $C$ meets the hyperplane $t=\tau$ (see Fig. 1, which illustrates the case $m=2$ ). Integrating (2.7) along $C$ gives

$$
U(P)=U(S)+\sum_{i=1}^{m} \int_{\tau}^{r+\Delta t}\left(\frac{\partial h}{\partial \lambda_{i}} l-A_{i}\right) U_{x,} d t .
$$

The coordinates of $\beta$ are determined by integrating the equations (2.5) so that

$$
\beta_{i}=\alpha_{i}-\int_{\tau}^{r+\Delta t} \frac{\partial h}{\partial \lambda_{i}} d t, \quad 1 \leqq i \leqq m .
$$

Equation (2.8) is our desired integral system.

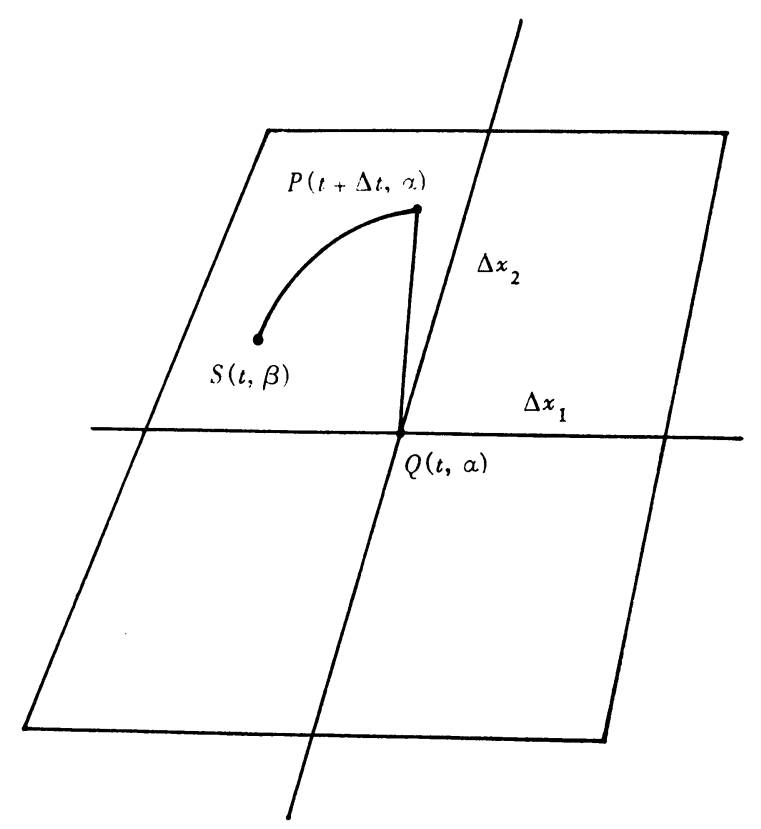

FIGURE 1 
3. Difference Schemes. We place a uniform rectangular mesh on $E^{m}$ in the usual way, using step sizes of $\Delta x_{1}, \cdots, \Delta x_{m}$ in the direction of $x_{1}, \cdots, x_{m}$, respectively. A step size of $\Delta t$ will be used for the time variable. Assuming we know values of $U$ at the time levels $t_{i}=j \Delta t, 0 \leqq j \leqq n$, we want to develop schemes which allow us to find values at $t_{n+1}=(n+1) \Delta t$.

The method we shall use to obtain such difference schemes will be to apply some quadrature formula to the integrals in Eqs. (2.8) and (2.9) and use an appropriate type of difference approximation for the space derivatives. For example, we could use Simpson's rule for the integrals along with a five-point central difference approximation for the derivatives. The resulting formula would be one of high-order accuracy. However, it would require values of $U$ at time $t_{n-1}$ as well as at $t_{n}$ and such "multilevel" methods are usually impractical from a computational point of view, especially for problems where $m>1$. Hence, while recognizing that such higher-order schemes can easily be generated, we shall confine our discussion to single-step methods.

3.1 Explicit Difference Scheme A. For the approximation of the integrals, we use

$$
\int_{t_{n}}^{t_{n+1}} f(t, \alpha) d t \doteqdot f\left(t_{n}, \alpha\right) \cdot \Delta t .
$$

This gives

$$
U(P) \div U(S)+\sum_{i=1}^{m}\left(\frac{\partial h}{\partial \lambda_{i}} I-A_{i}\right)_{0}\left(U_{x_{i}}\right)_{0} \Delta t,
$$

where $P, S$ and $Q$ are as in Fig. 1. Since $\beta$ is not, in general, a grid point, we approximate $U(S)$ by the truncated Taylor expansion about $Q=\left(t_{n}, \alpha\right)$,

$$
U(S)=U^{n}(\beta) \doteqdot U^{n}(\alpha)+\sum_{i}^{m}\left(\beta_{i}-\alpha_{i}\right)\left(U_{x_{i}}\right)_{0},
$$

where $U^{n}(\beta)=U\left(t_{n}, \beta\right)$. For the coordinates of $\beta$, we use (3.1) in (2.9) to get

$$
\beta_{i}=\alpha_{i}-\left(\partial h / \partial \lambda_{i}\right)_{Q} \Delta t,
$$

so that

$$
U(S) \doteqdot U(Q)-\sum_{1}^{m}\left(\frac{\partial h}{\partial \lambda_{i}}\right)_{0}\left(U_{x_{1}}\right)_{Q} \Delta t .
$$

Now, to approximate the space derivatives in (3.3), we use

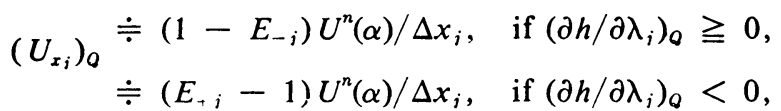

where $E_{+j}$ and $E_{-i}$ are, respectively, the forward and backward shift operators with respect to the space variable $x_{i}$. For the derivatives in (3.2), we use central differences

$$
\left(U_{x_{i}}\right)_{Q} \doteqdot\left(E_{+i}-E_{-i}\right) U^{n}(\alpha) / 2 \Delta x_{i} .
$$

Finally, our approximation for $U(P)$ is 


$$
\begin{aligned}
U(P)= & U^{n+1}(\alpha) \\
\doteqdot U^{n}(\alpha)+\sum_{i=1}^{n} \frac{\Delta t}{\Delta x_{i}}\left[-\left|\frac{\partial h}{\partial \lambda_{j}}\right|_{Q}+\right. & \frac{1}{2}\left(\left|\frac{\partial h}{\partial \lambda_{i}}\right| I-A_{i}\right)_{Q} E_{+i} \\
& \left.+\frac{1}{2}\left(\left|\frac{\partial h}{\partial \lambda_{j}}\right| I+A_{j}\right)_{0} E_{-i}\right] U^{n}(\alpha),
\end{aligned}
$$

which we shall call scheme $\mathrm{A}$.

Note that if we use $\left|\partial h / \partial \lambda_{i}\right|_{\theta}=1 / m r_{i}$, where $r_{i}=\Delta t / \Delta x_{i}$, then this scheme becomes the Lax scheme.

3.2 Implicit Difference Scheme B. If we use

$$
\int_{t_{n}}^{t_{n+1}} f(t, \alpha) d t \doteqdot \frac{\Delta t}{2}\left[f\left(t_{n+1}, \alpha\right)+f\left(t_{n}, \alpha\right)\right]
$$

as our integral approximation and the same derivative approximations as before, we get

$$
\begin{aligned}
& U(P)=U^{n+1}(\alpha) \\
& \doteqdot U^{n}(\alpha)+\sum_{j=1}^{m} \frac{\Delta t}{\Delta x_{i}}\left[\left.\cdot \frac{\partial h}{\partial \lambda_{i}}\right|_{0}\left(E_{ \pm i}-1\right)\right. \\
& \left.+\frac{1}{4}\left(\frac{\partial h}{\partial \lambda_{i}} I-A_{i}\right)_{Q}\left(E_{+i}-E_{-i}\right)\right] U^{n}(\alpha) \\
& +\frac{1}{4} \sum_{j=1}^{m} \frac{\Delta t}{\Delta x_{i}}\left[\left(\frac{\partial h}{\partial \lambda_{i}} I-A_{i}\right)_{Q}\left(E_{+j}-E_{-j}\right)\right] U^{n+1}(\alpha)
\end{aligned}
$$

which will be called scheme B. The operator $E_{ \pm i}$ is to be interpreted as $E_{+i}$ if $\left(\partial h / \partial \lambda_{j}\right)_{0}$ $<0$ and $E_{-j}$ if $\left(\partial h / \partial \lambda_{i}\right)_{0} \geqq 0$.

Since $U^{n+1}(\alpha)$ appears on both sides of (3.5), this is an implicit method and an iterative procedure is used to solve it. We can write the scheme as

$$
U^{n+1}(\alpha)=T U^{n+1}(\alpha)=C(\alpha)+S U^{n+1}(\alpha),
$$

where $C(\alpha)$ is independent of $U^{n+1}(\alpha)$. Then, with some initial guess $U_{0}^{n+1}(\alpha)$, we form successive iterates from

$$
U_{r+1}^{n+1}(\alpha)=T U_{r}^{n+1}(\alpha), \quad r=0,1,2, \cdots .
$$

To see when convergence of (3.7) can be guaranteed for arbitrary $U_{0}^{n+1}$, we use

THEOREM 1. The difference operator $T$ defined in (3.6) is a contraction operator provided that

(i) the $\lambda_{i}$ 's are chosen such that $\left|\partial h / \partial \lambda_{i}\right| \leqq\left\|A_{i}\right\|, 1 \leqq j \leqq m$, where $\left\|A_{j}\right\|^{2}=$ $\rho\left(A_{i}^{*} A_{i}\right)$;

(ii) the matrices $A_{i}$ are uniformly bounded by some constant $R$ for all $x$; and

(iii) $\Delta t, \Delta x_{i}$ are chosen such that

$$
\Delta t / \Delta x_{i}<1 / m R, \quad 1 \leqq j \leqq m .
$$

Proof. We first show that, for an arbitrary bounded vector function $F_{1}$ defined on $E^{m}, \phi_{1}=T F_{1}$ is also bounded. Let $\alpha$ be a lattice point. Then, from (3.6) and the 
Cauchy inequality, we have

$$
\begin{aligned}
\left|\phi_{1}(\alpha)\right|^{2} & =\phi_{1}^{*}(\alpha) \phi_{1}(\alpha) \leqq 2\left\{|C(\alpha)|^{2}+\frac{1}{16} \sum_{1}^{m}\left|r_{i}\left(\frac{\partial h}{\partial \lambda_{i}} I-A_{i}\right)\left[E_{+i}-E_{-i}\right] F_{1}(\alpha)\right|^{2}\right\} \\
& <2|C(\alpha)|^{2}+\frac{1}{m} \sum_{1}^{m}\left(\left|E_{+i} F_{1}(\alpha)\right|^{2}+\left|E_{-i} F_{1}(\alpha)\right|^{2}\right)
\end{aligned}
$$

Hence,

$$
\left\|\phi_{1}\right\|^{2}=\sum_{\alpha}|\phi(\alpha)|^{2} \cdot \Delta x<2\|C\|^{2}+2\left\|F_{1}\right\|^{2},
$$

where $\Delta x=\prod_{1}^{m} \Delta x_{i}$.

Next, if $F_{2}$ is also bounded and $\phi_{2}=T F_{2}$, then

$$
\begin{aligned}
\left|\phi_{1}(\alpha)-\phi_{2}(\alpha)\right|^{2} & =\left|\frac{1}{4} \sum_{1}^{m} r_{i}\left(\frac{\partial h}{\partial \lambda_{j}} I-A_{i}\right)\left[E_{+i}-E_{-i}\right]\left(F_{1}(\alpha)-F_{2}(\alpha)\right)\right|^{2} \\
& <\frac{1}{2 m} \sum_{1}^{m}\left\{\left|E_{+j}\left(F_{1}(\alpha)-F_{2}(\alpha)\right)\right|^{2}+\left|E_{-j}\left(F_{1}(\alpha)-F_{2}(\alpha)\right)\right|^{2}\right\},
\end{aligned}
$$

so that $\left\|\phi_{1}-\phi_{2}\right\|^{2}<\left\|F_{1}-F_{2}\right\|^{2}$. Q.E.D.

Using this result and the principle of contraction mappings, we see that, under the conditions (i)-(iii), the iteration (3.7) must converge to the unique solution of

$$
U^{n+1}=T U^{n+1}
$$

for any initial approximation $U_{0}^{n+1}$. We note in particular that, if we choose $U_{0}^{n+1}=U^{n}$, then $U_{1}^{n+1}$ is the approximation we would obtain for $U^{n+1}$ by applying scheme A to $U^{n}$. Hence, B will evidently give more accurate results, but at the expense of considerably more computation, since each iteration is approximately equivalent to a single step with A.

4. Convergence and Stability. We first consider the case where the coefficient matrices $A_{i}$ are constant. For scheme $\mathrm{A}$, we shall prove stability, provided certain conditions are satisfied. The convergence will then follow by the Lax Equivalence Theorem [14, p. 45]. Scheme $B$ will be shown to be stable whenever $A$ is.

We shall show stability by making use of the amplification matrix. Briefly, if we assume that $U$ is periodic in each space variable $x_{i}$ and has period $L_{i}$ in $x_{i}$, then the Fourier representation of $U^{n}(x)$ is

$$
U^{n}(x)=\left(\frac{1}{L_{1} L_{2} \cdots L_{m}}\right)^{1 / 2} \sum_{k \in \mathcal{L}} V^{n}(k) \exp (i k \cdot x),
$$

where $\mathfrak{L}$ is the lattice of points $k=\left(k_{1}, \cdots, k_{m}\right)$ such that each component $k_{i}$ runs over the values $2 \pi s_{i} / L_{i}, s_{i}$ an integer. From the difference scheme relationship between $U^{n}$ and $U^{n+1}$, we obtain the relation

$$
V^{n+1}(k)=G(\Delta t, k) V^{n}(k)
$$

between the Fourier coefficient vectors. $G(\Delta t, k)$ is called the amplification matrix. Denoting the eigenvalues of $G$ by $\nu_{i}, 1 \leqq i \leqq N$, the von Neumann necessary condition for stability is 


$$
\left|\nu_{i}\right| \leqq 1+O(\Delta t), \quad 1 \leqq i \leqq N,
$$

for all $k \in \mathcal{L}$ and $0<\Delta t<\tau$ for some $\tau>0$. Further, it can be shown [14] that, if $G(\Delta t, k)$ is uniformly diagonalizable, i.e., if for each $k$ there exists a nonsingular matrix $T$ such that $T^{-1} G T=\Lambda$ is diagonal and $T, T^{-1}$ are bounded independently of $k$ and $\Delta t$, then (4.1) is also sufficient for stability.

For scheme $\mathbf{A}$, the amplification matrix is

$$
\begin{aligned}
G(\Delta t, k)= & \left(1-\sum_{1}^{m}\left|\frac{\partial h}{\partial \lambda_{j}}\right| r_{i}\right) I+\frac{1}{2} \sum_{1}^{m}\left(\left|\frac{\partial h}{\partial \lambda_{j}}\right| I-A_{i}\right) r_{i} \exp \left(i k_{i} \Delta x_{i}\right) \\
& +\frac{1}{2} \sum_{1}^{m}\left(\left|\frac{\partial h}{\partial \lambda_{i}}\right| I+A_{i}\right) r_{i} \exp \left(-i k_{j} \Delta x_{i}\right),
\end{aligned}
$$

where $r_{i}=\Delta t / \Delta x_{i}$.

LeMmA 1. The amplification matrix $G(\Delta t, k)$, corresponding to scheme $\mathrm{A}$, is uniformly diagonalizable for all $k \in \mathcal{L}$ and for all $0<\Delta t<\tau$.

Proof. We can write the amplification matrix as

$$
G(\Delta t, k)=\left(1-2 \sum_{1}^{m}\left|\frac{\partial h}{\partial \lambda_{j}}\right| r_{j} \sin ^{2} \beta_{i}\right) I-i A,
$$

where $A=\sum A_{i} r_{i} \sin \alpha_{i}, \alpha_{i}=k_{i} \Delta x_{i}$ and $\beta_{i}=\alpha_{i} / 2$. Since the given system is hyperbolic, it follows that $A$ is uniformly diagonalizable and hence, so is $G(\Delta t, k)$.

With this result, we see that (4.1) is both necessary and sufficient for stability. Our next result gives sufficient conditions which guarantee that (4.1) holds.

Lemma 2. The eigenvalues of $G(\Delta t, k)$ for scheme A are uniformly bounded by unity in absolute value if

(i) There exists a constant $R>0$ such that $\left\|A_{j}\right\| \leqq R$ for all $x \in E^{m}$ and all $1 \leqq j \leqq m ;$

(ii) $\left|\partial h / \partial \lambda_{i}\right| \leqq R, 1 \leqq j \leqq m$; and

(iii) $r_{j} \leqq\left|\partial h / \partial \lambda_{j}\right| / m R^{2}, 1 \leqq j \leqq m$.

Proof. The eigenvalues of $G$ are of the form

$$
\nu=\left(1-2 \sum_{i}^{m} r_{j}\left|\frac{\partial h}{\partial \lambda_{j}}\right| \sin ^{2} \beta_{i}\right)-i \mu,
$$

where $\mu$ is an eigenvalue of $\sum A_{i} r_{i} \sin \alpha_{i}$ with $\alpha_{i}$ and $\beta_{i}$ as above. Then

$$
\begin{aligned}
|\nu|^{2} & =\left(1-2 \sum r_{i}\left|\frac{\partial h}{\partial \lambda_{j}}\right| \sin ^{2} \beta_{i}\right)^{2}+\mu^{2} \\
& \leqq 1-4 \sum r_{i}\left|\frac{\partial h}{\partial \lambda_{i}}\right| \sin ^{2} \beta_{i}+4 m \sum r_{i}^{2}\left|\frac{\partial h}{\partial \lambda_{i}}\right|^{2} \sin ^{4} \beta_{i}+\mu^{2} .
\end{aligned}
$$

Now,

$$
|\mu| \leqq\left\|\sum A_{i} r_{i} \sin \alpha_{i}\right\| \leqq R \sum r_{i}\left|\sin \alpha_{i}\right|,
$$

and so

$$
\mu^{2}=|\mu|^{2} \leqq 4 m R^{2} \sum r_{i}^{2}\left(\sin ^{2} \beta_{i}-\sin ^{4} \beta_{i}\right)
$$

Hence, 


$$
\begin{aligned}
|\nu|^{2} \leqq & +4 \sum r_{i}\left(m R^{2} r_{i}-\left|\frac{\partial h}{\partial \lambda_{j}}\right|\right) \sin ^{2} \beta_{i} \\
& +4 m \sum r_{i}^{2}\left(\left|\frac{\partial h}{\partial \lambda_{i}}\right|^{2}-R^{2}\right) \sin ^{4} \beta_{i} \leqq 1 . \quad \text { Q.E.D. }
\end{aligned}
$$

THEOREM 2. If the $\lambda_{i}$ 's are chosen so that

$$
\left|\partial h / \partial \lambda_{j}\right| \leqq \max _{i}\left\|A_{i}\right\|=\bar{R}, \quad 1 \leqq j \leqq m,
$$

then, in the case of constant coefficients in (1.1), difference scheme $\mathrm{A}$ is stable and convergent if

$$
r_{i} \leqq\left|\partial h / \partial \lambda_{i}\right| / m \bar{R}^{2}, \quad 1 \leqq j \leqq m .
$$

Proof. By Lemma 2, we see that the eigenvalues of $G(\Delta t, k)$ will satisfy the von Neumann stability condition (4.1). The scheme is easily shown to be consistent [12] and so convergence follows from the Lax Equivalence Theorem. Q.E.D.

For scheme B, we prove

THEOREM 3. If the conditions of Theorem 2 hold with strict inequality in (4.2), then scheme $\mathrm{B}$ is also stable for problems with constant coefficients.

Proof. We shall use the notation $U^{n}$ and $W^{n}$ for the approximations by schemes $\mathrm{A}$ and $\mathrm{B}$, respectively, to the solution at time $t=n \Delta t$. First, we note that, by hypothesis, the operator $T$ of Theorem 1 is a contraction operator. Hence, the vectors $W_{r}^{n+1}$, defined by

$$
W_{r+1}^{n+1}=T W_{r}^{n+1}, \quad r \geqq 0,
$$

converge to $W^{n+1}$ for arbitrary initial guess $W_{0}^{n+1}$. In particular, if we set $W_{0}^{n+1}=U^{n}$, then, as we observed above, $W_{1}^{n+1}=U^{n+1}$, and so we have, for any $r$,

$$
\left\|W_{r+1}^{n+1}-W_{r}^{n+1}\right\|=\left\|T\left(W_{r}^{n+1}-W_{r-1}^{n+1}\right)\right\| \leqq \alpha^{r}\left\|U^{n+1}-U^{n}\right\|,
$$

where $0<\alpha<1$. Now,

$$
\begin{aligned}
\left\|W_{r+1}^{n+1}-U^{n+1}\right\| & =\left\|W_{r+1}^{n+1}-W_{1}^{n+1}\right\| \\
& \leqq\left\|W_{r+1}^{n+1}-W_{r}^{n+1}\right\|+\cdots+\left\|W_{2}^{n+1}-W_{1}^{n+1}\right\| \\
& =\alpha \frac{1-\frac{\alpha^{r}}{1-}}{\alpha}\left\|U^{n+1}-U^{n}\right\| .
\end{aligned}
$$

Hence,

$$
\left\|W_{r+1}^{n+1}\right\| \leqq\left\|U^{n+1}\right\|+\alpha \frac{1-\alpha^{r}}{1-\alpha}\left\|U^{n+1}-U^{n}\right\|
$$

which is uniformly bounded for all $n$ and all $r$. The result then follows from the inequality

$$
\left\|W^{n+1}\right\| \leqq\left\|W_{r+1}^{n+1}\right\|+\left\|W^{n+1}-W_{r+1}^{n+1}\right\| \leqq\left\|W_{r+1}^{n+1}\right\|+\epsilon,
$$

for arbitrary $\epsilon>0$, provided $r$ is sufficiently large. Q.E.D.

For problems with variable coefficients, we can show local stability holds under conditions similar to those of Theorem 2. We have, without proof, 
THEOREM 4. Suppose that (1.1) is quasi-linear and that the coefficient matrices $A_{i}=A_{i}(U, x)$ are uniformly bounded. If the $\lambda_{i}$ 's are chosen so that

$$
\left|\partial h / \partial \lambda_{j}\right| \leqq \max _{i}\left\|A_{i}\right\|=\bar{R}, \quad 1 \leqq j \leqq m,
$$

then scheme $\mathrm{A}$ is locally stable if

$$
r_{i} \leqq\left|\partial h / \partial \lambda_{i}\right| / m \bar{R}^{2}, \quad 1 \leqq j \leqq m
$$

Local stability of scheme B follows from a result similar to Theorem 3 . We omit the details.

In case the $A_{i}$ 's in (1.1) are symmetric, we can use the theory of Kreiss [8] on dissipative schemes to show global stability.

Definition. A difference scheme is dissipative of order $2 r, r$ a positive integer, if there exists a constant $\delta>0$ such that for any $\xi=\left(\xi_{1}, \cdots, \xi_{m}\right), \xi_{i}=k_{i} \Delta x_{i}$, satisfying $\max _{j}\left|\xi_{j}\right| \leqq \pi$, and all $\Delta t$ less than some $\tau>0$,

$$
|\nu| \leqq 1-\delta|\xi|^{2 r}, \text { for all } x,
$$

where $\nu=\nu(x, \Delta t, \xi)$ is an eigenvalue of the local amplification matrix $G(x, \Delta t, \xi)$.

THEOREM 5. Suppose the system (1.1) of differential equations is symmetric and that the coefficient matrices $A_{i}$ are uniformly bounded by a constant $\bar{R}$ and uniformly Lipschitz continuous functions of $x$, then the explicit difference scheme $\mathbf{A}$ is stable if, in Lemma 2, condition (ii) and condition (iii) with strict inequality are satisfied.

Proof. According to Kreiss' theorem, we need to show that scheme A is dissipative of order 2 since it is accurate of order 1 . Under our hypotheses, we can show by an argument like that of Lemma 2 that, for all $x$ and all $\xi$ with $\left|\xi_{i}\right| \leqq \pi, 1 \leqq j \leqq m$, there exists a $\delta>0$ such that

$$
|\nu(x, t, \xi)|^{2}<1-\delta|\xi|^{2},
$$

from which it follows that scheme $\mathbf{A}$ is dissipative of order 2. Q.E.D.

5. Choice of Bicharacteristics. It is natural to ask if the choice of different bicharacteristics makes any significant difference in the solution obtained. To this end, let us examine the difference scheme A corresponding to two different choices of bicharacteristics. This may be done by choosing different eigenvalues $h$ of the matrix $\sum A_{i} \lambda_{i}$ and/or different sets of values for the $\lambda_{i}$ 's. In any case, the quantities $\left|\partial h / \partial \lambda_{j}\right|$ occurring in the difference scheme will have different values for different choices. Let $a_{j}$ and $a_{j}^{\prime}, 1 \leqq j \leqq m$, be the two sets of values of $\left|\partial h / \partial \lambda_{j}\right|$. Then, denoting the solutions at time $t=(n+1) \Delta t$ corresponding to $a_{j}$ 's and $a_{j}^{\prime}$ 's by $U^{n+1}$ and $W^{n+1}$, respectively, and assuming we use the same value $U^{n}$ at time $t=n \Delta t$, we can write

$$
\begin{aligned}
U^{n+1}(x)-W^{n+1}(x) & =\sum\left(a_{i}^{\prime}-a_{j}\right) r_{j}\left[U^{n}(x)-\frac{1}{2}\left(E_{+j} U^{n}(x)+E_{-j} U^{n}(x)\right)\right] \\
& =\sum\left(a_{i}^{\prime}-a_{i}\right) r_{j} O\left(\Delta x_{j}^{2}\right)=O\left(\Delta t^{2}\right) .
\end{aligned}
$$

The latter equality follows from the stability condition $r_{i} \leqq\left|\partial h / \partial \lambda_{j}\right| / m R^{2}, 1 \leqq j \leqq m$, for then $\Delta t$ and the $\Delta x_{j}$ 's are of the same order of magnitude and $\left(a_{i}^{\prime}-a_{i}\right) r_{i}=O(1)$.

Hence, the discrepancy in the solutions obtained by using two different bichar- 
acteristics is at most of the same order as the truncation error, and so, up to the order of accuracy maintained, it does not matter if one or another of the bicharacteristics is used.

6. Numerical Results. We present the results of computations on three specific problems. The problems were chosen not only to illustrate the implementation of our methods but also to demonstrate their usefulness in solving complicated problems such as the third example, where $m=3$. Computations were done on an IBM 360/65 using single precision.

(1) Two-Dimensional Wave Equation. The differential system is

$$
\frac{\partial U}{\partial t}=\left[\begin{array}{llc}
0 & 0 & 0 \\
0 & 0 & 1 \\
0 & \frac{1}{2} & 0
\end{array}\right] \frac{\partial U}{\partial x}+\left[\begin{array}{rrr}
0 & 0 & -1 \\
0 & 0 & 0 \\
-\frac{1}{2} & 0 & 0
\end{array}\right] \frac{\partial U}{\partial y},
$$

with initial conditions

$$
U(0, x, y)=\left[\begin{array}{c}
\cos x \cos y \\
\sin x \sin y \\
0
\end{array}\right]
$$

We have

$$
H=\lambda\left(\lambda^{2}-\frac{1}{2}\left(\lambda_{1}^{2}+\lambda_{2}^{2}\right)\right)
$$

so that

$$
h_{1}=\left(\frac{\lambda_{1}^{2}+\lambda_{2}^{2}}{2}\right)^{1 / 2}, \quad h_{2}=-h_{1}, \quad h_{3}=0
$$

and

$$
\frac{\partial h_{1}}{\partial \lambda_{1}}=-\frac{\partial h_{2}}{\partial \lambda_{1}}=\frac{\lambda_{1}}{\left(2\left(\lambda_{1}^{2}+\lambda_{2}^{2}\right)\right)^{1 / 2}}, \quad \frac{\partial h_{1}}{\partial \lambda_{2}}=-\frac{\partial h_{2}}{\partial \lambda_{2}}=\frac{\lambda_{2}}{\left(2\left(\lambda_{1}^{2}+\lambda_{2}^{2}\right)\right)^{1 / 2}}
$$

The exact solution is

$$
U=\left[\begin{array}{lll}
\cos x & \cos y & \cos t \\
\sin x & \sin y & \cos t \\
\cos x & \sin y & \sin t
\end{array}\right]
$$

Computations were done using each of scheme A, scheme $\mathbf{B}$ and the general scheme of Lax [9]. In each case, we used $\Delta x=\Delta y=.1$ and $r_{1}=r_{2}=.1$. For scheme B, we used only two iterations of (3.7) per time step. In Table I, we give values obtained by each method as well as the true value at $x=y=.5$ at various time levels. Computing times for 100 time steps were $.26, .48$ and .32 minute for the respective methods.

The results for method $\mathrm{B}$ are, of course, the best, but were obtained at approximately double the computing cost, which, as indicated above, was to be expected. This behaviour was observed in all our computations. 
TABLE I

Solution of Wave Equation at $x=y=.5$. (For Numerical Computations

$$
\Delta x=\Delta y=.1, \Delta t / \Delta x=.1 \text {.) }
$$

\begin{tabular}{llccc}
\hline Time $t$ & Solution & $u$ & $v$ & $w$ \\
\hline \multirow{3}{*}{0.2} & Exact & 0.7548 & 0.2253 & 0.0836 \\
& Method A & 0.7481 & 0.2233 & 0.0827 \\
& Method B & 0.7552 & 0.2256 & 0.0831 \\
& Lax & 0.6925 & 0.2067 & 0.0779 \\
\hline \multirow{3}{*}{0.5} & Exact & 0.6759 & 0.2017 & 0.2017 \\
& Method A & 0.6616 & 0.1974 & 0.1975 \\
& Method B & 0.6778 & 0.2026 & 0.2007 \\
& Lax & 0.5904 & 0.1762 & 0.1822 \\
\hline \multirow{3}{*}{1.0} & Exact & 0.4226 & 0.1261 & 0.3517 \\
& Method A & 0.4079 & 0.1217 & 0.3432 \\
& Method B & 0.4286 & 0.1281 & 0.3527 \\
& Lax & 0.3584 & 0.1069 & 0.3124 \\
\hline \multirow{3}{*}{2.0} & Exact & -0.3205 & -0.0956 & 0.3826 \\
& Method A & -0.3169 & -0.0946 & 0.3762 \\
& Method B & not computed & not computed & not computed \\
& Lax & -0.2911 & -0.0869 & 0.3340 \\
\hline
\end{tabular}

The remaining problems are of mixed initial-boundary-value type. Although we have only discussed solving the pure initial-value problem, it is quite easy to modify the difference schemes to incorporate boundary conditions. One such modification would be to restrict the choice of bicharacteristic to ensure that the point $S$ in Fig. 1 lies within the region of interest. Also, for approximating space derivatives at boundary points, we must use forward or backward differences (whichever is applicable) for derivatives with components normal to the boundary. We shall not discuss the stability properties of the resulting system of difference equations.

(2) Plane Unsteady Motion of an Isentropic Inviscid Fluid. The system of equations is

where

$$
\frac{\partial U}{\partial t}+A \frac{\partial U}{\partial x}+B \frac{\partial U}{\partial y}=0
$$

$$
U=\left[\begin{array}{c}
\rho \\
u \\
v \\
p
\end{array}\right], \quad A=\left[\begin{array}{cccc}
u & \rho & 0 & 0 \\
0 & u & 0 & \rho^{-1} \\
0 & 0 & u & 0 \\
0 & \rho c^{2} & 0 & u
\end{array}\right], \quad B=\left[\begin{array}{cccc}
v & 0 & \rho & 0 \\
0 & v & 0 & 0 \\
0 & 0 & v & \rho^{-1} \\
0 & 0 & \rho c^{2} & v
\end{array}\right] \text {. }
$$


Here, $\rho$ represents the density, $p$ the pressure and $u, v$ are the components of velocity in the $x$ and $y$ directions respectively, $c$ is the local sound speed given by $c^{2}=\gamma p / \rho$, where $\gamma$ is the adiabatic constant for the fluid.

The problem was solved in the unit square $0 \leqq x, y \leqq 1$ with $\gamma=2$, subject to the initial conditions,

$$
\begin{aligned}
& \rho(0, x, y)=(2-x)(2-y) / 10, \\
& p(0, x, y)=\rho^{2}(0, x, y), \\
& u(0, x, y)=v(0, x, y)=0,
\end{aligned}
$$

and boundary conditions

$$
u(t, 0, y)=u(t, 1, y)=v(t, x, 0)=v(t, x, 1)=0, \quad t>0 .
$$

The eigenvalues for the matrix $A \lambda_{1}+B \lambda_{2}$ are

$$
\begin{aligned}
& h_{1}=u \lambda_{1}+v \lambda_{2}, \\
& h_{2}=u \lambda_{1}+v \lambda_{2}+c\left(\lambda_{1}^{2}+\lambda_{2}^{2}\right)^{1 / 2}, \\
& h_{3}=u \lambda_{1}+v \lambda_{2}-c\left(\lambda_{1}^{2}+\lambda_{2}^{2}\right)^{1 / 2} .
\end{aligned}
$$

Solutions were obtained using each of the $h_{i}$ 's in scheme $\mathrm{A}$ and the results were the same up to four decimal places. We also used the generalized Lax-Wendroff twostep method [9] which is a well-tested method for such problems. Table II shows some

TABLE II

Solution of Plane Unsteady Hydrodynamic Motion Problem at $x=y=.5 .(\Delta x=$ $\Delta y=.1, \Delta t / \Delta x=.4$ for Method A, $\Delta t / \Delta x=.2$ for Lax-Wendroff Two-Step Method.)

\begin{tabular}{llcccc}
\hline Time $t$ & Solution & $\rho$ & $u$ & $v$ & $p$ \\
\hline \multirow{2}{*}{04} & Method A & 0.2250 & 0.0120 & 0.0120 & 0.0507 \\
& Lax-Wendroff & 0.2250 & 0.0120 & 0.0120 & 0.0507 \\
\hline \multirow{2}{*}{0.2} & Method A & 0.2266 & 0.0604 & 0.0604 & 0.0518 \\
& Lax-Wendroff & 0.2268 & 0.0604 & 0.0604 & 0.0514 \\
\hline \multirow{2}{*}{0.6} & Method A & 0.2306 & 0.1638 & 0.1639 & 0.0540 \\
& Lax-Wendroff & 0.2331 & 0.1694 & 0.1694 & 0.0542 \\
\hline \multirow{2}{*}{1.0} & Method A & 0.2013 & 0.1831 & 0.1832 & 0.4105 \\
& Lax-Wendroff & 0.2136 & 0.1901 & 0.1901 & 0.4548 \\
\hline
\end{tabular}

typical results. It can be seen that our results compare favourably with those obtained by Lax-Wendroff. Comparative computing times were about .75 and 1.0 minute, respectively, for scheme A and Lax-Wendroff. 
(3) Magnetohydrodynamics Initial-Pressure Problem. This problem was considered by Friedlander [5]. It is a problem in three space dimensions and concerns the propagation of small disturbances in a compressible fluid, which is also a conductor of electricity, in the presence of a uniform magnetic field. It is assumed that the fluid fills the whole space. Energy dissipation by viscosity, heat conduction and Joule heat is neglected as is the displacement current. By linearizing the Lundquist equations, Friedlander obtained the system

$$
\begin{aligned}
\rho_{0} \frac{\partial v}{\partial t}+\operatorname{grad} p^{\prime}+\frac{\mu}{4 \pi} H_{0} \times \operatorname{curl} H^{\prime} & =0, \\
\frac{\partial p^{\prime}}{\partial t}+\rho_{0} \operatorname{div} v & =0, \\
\frac{\partial H^{\prime}}{\partial t}-\operatorname{curl}\left(v \times H_{0}\right) & =0, \\
\operatorname{div} H^{\prime} & =0,
\end{aligned}
$$

where $p^{\prime}=p-p_{0}, H^{\prime}=H-H_{0}, v$ is the velocity and $\rho$ is the density. Choosing the direction of $H_{0}$ as the $x$-axis, we get

$$
\begin{aligned}
\frac{\partial v_{x}}{\partial t}+\frac{1}{\rho_{0}} \frac{\partial p^{\prime}}{\partial x} & =0 \\
\frac{\partial v_{\nu}}{\partial t}+\frac{1}{\rho_{0}} \frac{\partial p^{\prime}}{\partial y}+\frac{\mu H_{0}}{4 \pi \rho_{0}}\left(\frac{\partial H_{x}^{\prime}}{\partial y}-\frac{\partial H_{y}^{\prime}}{\partial x}\right) & =0 \\
\frac{\partial v_{z}}{\partial t}+\frac{1}{\rho_{0}} \frac{\partial p^{\prime}}{\partial z}+\frac{\mu H_{0}}{4 \pi \rho_{0}}\left(\frac{\partial H_{x}^{\prime}}{\partial z}-\frac{\partial H_{z}^{\prime}}{\partial x}\right) & =0 \\
\frac{\partial p^{\prime}}{\partial t}+c^{2} \rho_{0}\left(\frac{\partial v_{x}}{\partial x}+\frac{\partial v_{y}}{\partial v}+\frac{\partial v_{z}}{\partial z}\right) & =0 \\
\frac{\partial H_{x}}{\partial t}+H_{0}\left(\frac{\partial v_{y}}{\partial y}+\frac{\partial v_{z}}{\partial z}\right) & =0 \\
\frac{\partial H_{y}}{\partial t}-H_{0} \frac{\partial v_{y}}{\partial x} & =0 \\
\frac{\partial H_{z}}{\partial t}-H_{0} \frac{\partial v_{z}}{\partial x} & =0
\end{aligned}
$$

where $p^{\prime}=c^{2} \rho^{\prime}, v_{x}$ is the $x$-component of velocity and so on. The initial conditions are

$$
\begin{aligned}
v(0, x, y, z) & =0, \\
p^{\prime}(0, x, y, z) & =F(x \cos \theta+y \sin \theta \cos \phi+z \sin \theta \sin \phi), \\
H^{\prime}(0, x, y, z) & =0
\end{aligned}
$$

where $F$ is an arbitrary function. The exact solution to this problem is given by Friedlander [5]. 
R. L. JOHNSTON AND S. K. PAL

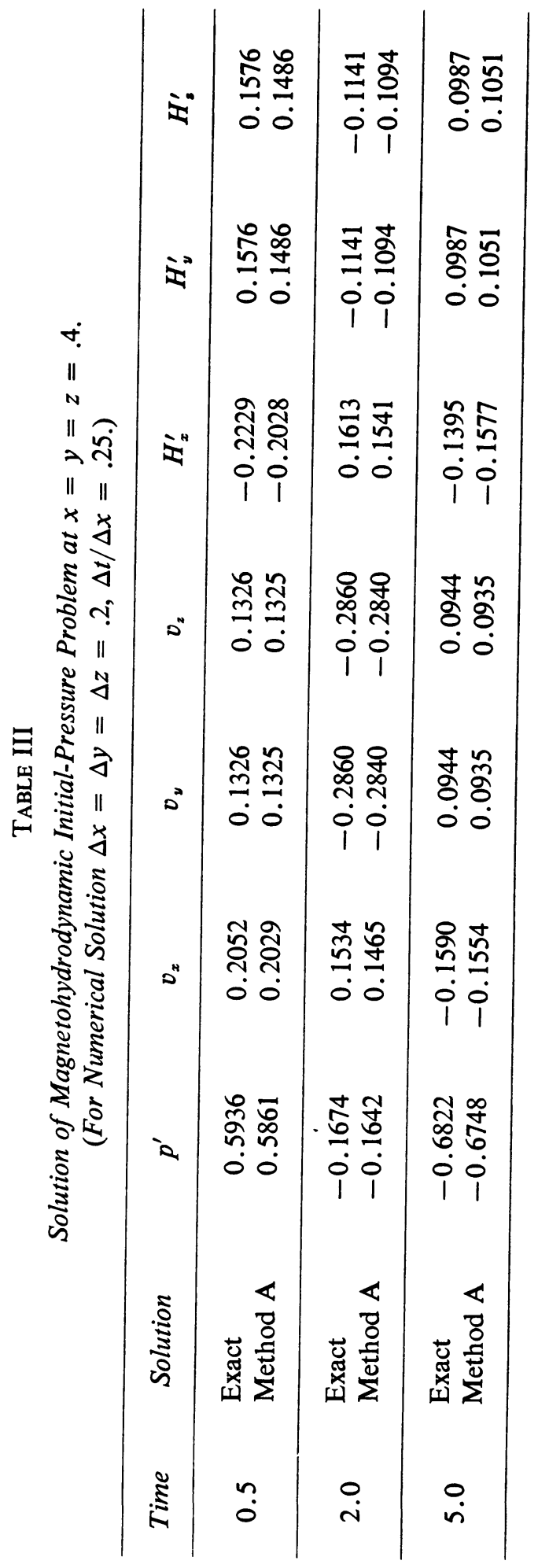


The eigenvalues of $A=\sum A_{i} \lambda_{i}$ are $0, \pm c_{f}, \pm c_{s}, \pm b_{x}$, where

$$
\begin{aligned}
& c_{f}^{2}=\frac{1}{2}\left\{\left(c^{2}+b^{2}\right) \lambda^{2}+\lambda\left(\left(c^{2}+b^{2}\right)^{2} \lambda^{2}-4 c^{2} b^{2} \lambda_{1}^{2}\right)^{1 / 2}\right\}, \\
& c_{s}^{2}=\frac{1}{2}\left\{\left(c^{2}+b^{2}\right) \lambda^{2}-\lambda\left(\left(c^{2}+b^{2}\right)^{2} \lambda^{2}-4 c^{2} b^{2} \lambda_{1}^{2}\right)^{1 / 2}\right\}, \\
& b_{x}^{2}=\mu H_{0}^{2} \lambda_{1}^{2} / 4 \pi \rho_{0},
\end{aligned}
$$

with $\lambda^{2}=\lambda_{1}^{2}+\lambda_{2}^{2}+\lambda_{3}^{2}$ and $b^{2}=H_{0}^{2} / 4 \pi \rho_{0}$.

The region of interest was taken to be the unit cube $0 \leqq x, y, z \leqq 1$. Boundary conditions were derived from the known exact solution. We used $F(\alpha)=\cos \alpha$, $H_{0}=5, \mu=1, \rho_{0}=1, \theta=\phi=\pi / 4$. The explicit scheme A was used with the eigenvalues corresponding to $c_{f}$ and $c_{.}$. Mesh sizes used were $\Delta x=\Delta y=\Delta z=.2$, $\Delta t=.05$. Some typical results are given in Table III. These results are not as accurate as in the previous examples and this is due to the rather coarse mesh used. Computation time was about 6 minutes and would, of course, be greatly increased for finer meshes since we are working with three space dimensions.

7. Concluding Remarks. We have described and analyzed two finite difference schemes which can be used to solve a first-order quasi-linear hyperbolic system of partial differential equations. They have the advantage that they are applicable to problems in an arbitrary number of space dimensions and are relatively easy to implement. One possible significant disadvantage is that one must know an explicit representation for at least one of the eigenvalues $h_{i}$. However, in most practical problems, these eigenvalues are usually known beforehand and so this difficulty will not be encountered very often.

We conclude by remarking that we tried our methods on a hydrodynamic problem involving shock [12]. The numerical solutions obtained all had a well-defined shock front but the shock velocity was incorrect. This sort of behaviour is typical of many methods when applied to such problems and our methods apparently have not escaped these troubles. We therefore do not recommend our methods for shock problems.

Department of Computer Science

University of Toronto

Toronto, Canada

Department of Mathematics

Laurentian University

Sudbury, Ontario, Canada

1. S. D. BAXTER, Numerical Methods for the Solution of Hyperbolic Differential Equations with the Aid of Electronic Computers, Ph.D. Thesis, University of Toronto, Toronto, Ont., 1958.

2. D. S. BUTLER, "The numerical solution of hyperbolic systems of partial differential equations in three independent variables," Proc. Roy. Soc. London Ser. A, v. 255, 1960, pp. 232-252. MR 22 \#10193.

3. R. Courant, E. Isaacson \& M. REes, "On the solution of non-linear hyperbolic differential equations by finite differences," Comm. Pure Appl. Math., v. 5, 1952, pp. 243-255.

4. G. F. D. Duff, Partial Differential Equations, Math. Expositions, no. 9, Univ. of Toronto Press, Toronto, 1956. MR 17, 1210.

5. F. G. Friedlander, "Sound pulses in a conducting medium," Proc. Cambridge Philos. Soc., v. 55, 1959, pp. 341-367. MR 22 \#1289. 
6. D. R. HARTree, Some Practical Methods of Using Characteristics in the Calculation of Non-Steady Compressible Flow, Report LA-HU-1, Harvard University, Cambridge, Mass., 1953.

7. A. Jeffréy \& T. TANiuti, Non-Linear Wave Propagation. With Applications to Physics and Magnetohydrodynamics, Academic Press, New York, 1964. MR 29 \#4410.

8. H. O. KREISs, "On difference approximations of the dissipative type for hyperbolic differential equations," Comm. Pure Appl. Math., v. 17, 1964, pp. 335-353. MR 29 \#4210.

9. P. D. LAX, "Differential equations, difference equations and matrix theory," Comm. Pure Appl. Math., v. 11, 1958, pp. 175-194. MR $20 \# 4572$.

10. P. D. LaX \& B. WENDROFF, "Systems of conservation laws," Comm. Pure Appl. Math., v. 13, 1960, pp. 217-237. MR $22 \# 11523$.

11. P. D. LAX \& B. WENDROFF, "Difference schemes for hyperbolic equations with high order of accuracy," Comm. Pure Appl. Math., v. 17, 1964, pp. 381-398. MR 30 \#722.

12. S. K. PAL, Numerical Solution of First-Order Hyperbolic Systems of Partial Differential Equations, Ph.D. Thesis, University of Toronto, 1969; Technical Report \#13, Department of Computer Science, University of Toronto, Toronto, Ontario.

13. A. RAlston \& H. S. Wilf (Editor), Mathematical Methods for Digital Computers. Vol. I, Wiley, New York, 1960. MR 22 \#8680.

14. R. D. Richtmyer \& K. W. Morton, Difference Methods for Initial-Value Problems, 2nd ed., Interscience Tracts in Pure and Appl. Math., no. 4, Interscience, New York, 1967. MR 36 \#3515.

15. W. G. Strang, "Accurate partial difference methods. II. Non-linear problems," Numer. Math., v. 6, 1964, pp. 37-46. MR $29 \# 4215$. 